\title{
Patient with Acute Myocardial Infarction and Leriche Syndrome
}

\author{
Ana Vujaklija Brajković, Ivan Gornik, Ozrenka Zlopaša, Nina Gubarev Vrdoljak, \\ Radovan Radonić and Vladimir Gašparović
}

Key words: myocardial infarction, Leriche syndrome, smoking

(Inter Med 49: 349-350, 2010)

(DOI: 10.2169/internalmedicine.49.2978)

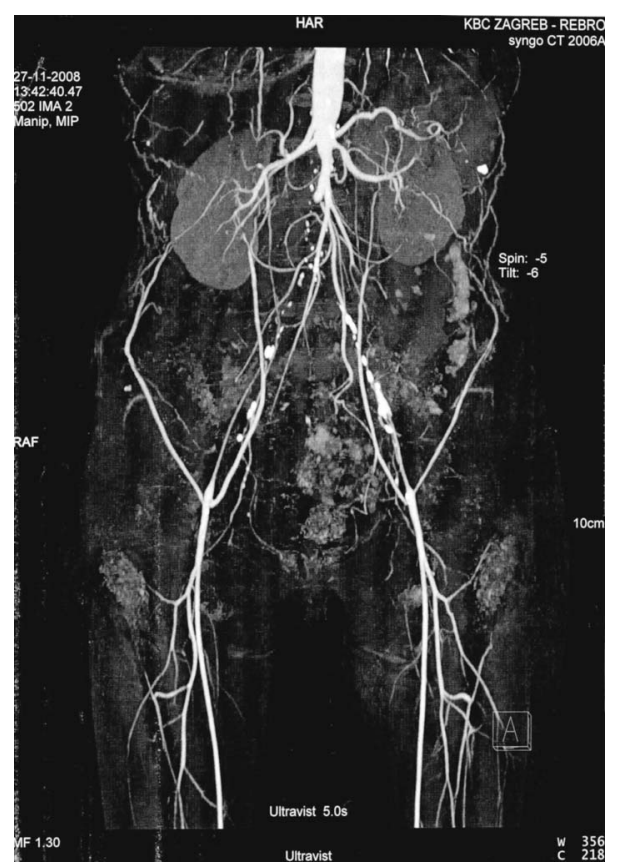

Picture 1. MSCT angiography showed partial thrombosis of the aorta between renal arteries and the inferior mesenteric artery and complete thrombosis of the aorta distally with development of collateral blood vessels. Stenosis of the left renal artery was also present.

A 39-year-old woman with acute onset of chest pain came to the emergency department. Diagnosis of myocardial infarction with ST segment elevation and complete atrioventricular block was made. Primary percutaneous coronary intervention was performed through the radial artery due to absent pulsations of femoral arteries. Occlusion of the proximal right coronary artery was found and a stent was implanted. The left coronary artery had no stenotic lesions. MSCT angiography showed partial thrombosis of the aorta between renal arteries and inferior mesenteric artery and complete thrombosis distally with already developed arterial collaterals, and revascularization on the level of femoral arteries (Picture 1). The ascending aorta, aortic arch, brachiocephalic artery, left common carotid artery, left subclavian artery and descending thoracic aorta showed no narrowing or occlusion. History of fatigue, weight loss, low-grade fever, arthralgias or myalgias, neurologic symptoms or syncope and hypertension were all negative. Pulsation was present in both brachial arteries. The value of systemic blood pressure was even in both arms. No bruit was heard over 
subcalvian arteries. Laboratory tests were performed to investigate advanced atherosclerosis. Triglycerides and cholesterol were normal. Acute phase reactants (erythrocyte sedimentation rate, serum C-reactive protein and alpha-2 globulin concentrations) were also normal. Screening for thrombophilia (including AT III, proteins $\mathrm{C}$ and S, plasminogen, fibrinogen, factor V Leiden, prothrombin 20210A polymorphism, MTHFR mutation, homocistein and antiphospholipid antibodies-lupus anti-coagulant, b2-glycoprotein I and anticardiolipin antibodies) and autoimmune disorders (including complement, cryoglobulins, antinuclear, antineutrophil cytoplasmic, anti-DNA and antiphospholipid antibodies) were negative. Smoking was proven to be the only risk factor for advanced atherosclerosis in this patient. A few months later the patient underwent aortobifemoral bypass surgery, quit smoking and felt better.

(C) 2010 The Japanese Society of Internal Medicine http://www.naika.or.jp/imindex.html 